Recma

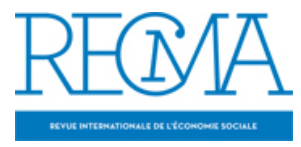

\title{
Une régulation de l'hybridation entre marchand et non-marchand
}

\author{
Les cas des formes de production de logiciels libres
}

A "regulation" of hybridization between market and non-market: the case of free software production forms

\section{Florian Perret et Martino Nieddu}

Numéro 304, mai 2007

URI : https://id.erudit.org/iderudit/1021532ar

DOI : https://doi.org/10.7202/1021532ar

Aller au sommaire du numéro

Éditeur(s)

Association Recma

ISSN

1626-1682 (imprimé)

2261-2599 (numérique)

Découvrir la revue

Citer cet article

Perret, F. \& Nieddu, M. (2007). Une régulation de l'hybridation entre marchand et non-marchand : les cas des formes de production de logiciels libres. Revue internationale de l'économie sociale, (304), 63-75.

https://doi.org/10.7202/1021532ar
Résumé de l'article

L'article s'intéresse aux communautés de production de logiciels libres, pour montrer la persistance de trois types de patrimoines articulés : communs, collectifs ou privés. Si le logiciel libre était à l'origine basé sur une production coopérative non marchande, son succès va provoquer une arrivée massive d'acteurs marchands. Les communautés vont chercher à pérenniser leurs valeurs de coopération et de partage du savoir, au travers de règles portées par des cadres juridiques et par la création d'associations. L'économie sociale apparaît alors comme un dispositif au coeur de la régulation économique du secteur, car elle met en forme des règles d'articulation du marchand et du non-marchand. Les auteurs constatent que ces régulations non seulement permettent la préservation et l'extension du patrimoine commun que constituent les logiciels libres, mais aussi influent sur l'ensemble du marché des logiciels, libres ou non, en obligeant les acteurs lucratifs à se repositionner et à faire évoluer leurs stratégies pour y intégrer une part de non-marchand. 


\section{UNE RÉGULATION DE L'HYBRIDATION ENTRE MARCHAND ET NON-MARCHAND} Le cas des formes de production de logiciels libres

(*) F. Perret et M. Nieddu sont respectivement allocataire de recherche et maître de conférences à l'université de Reims ChampagneArdenne, et tous deux membres de son laboratoire "Organisations marchandes et institutions " (OMI). La communication utilise, outre la littérature économique, divers matériaux, notamment des interviews et des relevés de discussions au sein de communautés, sur sites Internet, recensées par Florian Perret (florian.perret@univ-reims.fr).

\author{
par Florian Perret et Martino Nieddu ${ }^{(*)}$
}

L'article sintéresse aux communautés de production de logiciels libres, pour montrer la persistance de trois types de patrimoines articulés: communs, collectifs ou privés. Si le logiciel libre était à l'origine basé sur une production coopérative non marchande, son succès va provoquer une arrivée massive d'acteurs marchands. Les communautés vont chercher à pérenniser leurs valeurs de coopération et de partage du savoir, au travers de règles portées par des cadres juridiques et par la création d'associations. L'économie sociale apparaît alors comme un dispositif au coeur de la régulation économique du secteur, car elle met en forme des règles d'articulation $d u$ marchand et du non-marchand. Les auteurs constatent que ces régulations non seulement permettent la préservation et l'extension du patrimoine commun que constituent les logiciels libres, mais aussi influent sur l'ensemble du marché des logiciels, libres ou non, en obligeant les acteurs lucratifs à se repositionner et à faire évoluer leurs stratégies pour y intégrer une part de non-marchand.
(1) Dans cet article, nous désignerons comme relevant de l'économie sociale les pratiques informelles de production de logiciels libres d'avant 1984, le travail bénévole ou non des producteurs de logiciels libres après 1984 et la production organisée par les fondations à but non lucratif.

(2) Dans la licence dite GPL (General Public License), Richard Stallman définit les logiciels libres en détournant le copyright tout en se basant sur celui-ci. II y ajoute des spécificités qui permettent de protéger à la fois l'auteur et l'œuvre, qui apparaît ainsi comme un bien commun. Il la présente aux Français en 2006 en utilisant une analogie avec "Liberté, égalité, fraternité " pour expliciter les spécificités de cette licence:

- liberté d'accès au code source du logiciel pour l'étudier, le modifier et le distribuer;

- égalité d'accès au code source et absence de discrimination pour un

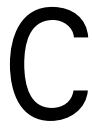
omme elle l'a fait à d'autres périodes et pour d'autres secteurs, l'économie sociale ${ }^{(1)}$ joue un rôle décisif dans la structuration de l'industrie du logiciel (Prades, 2004b). En effet, les logiciels libres, qui sont aujourd'hui au cœur de cette industrie, relèvent de l'économie sociale, d'abord dans les pratiques, puis dans la mise en place de règles et d'institutions formelles. On situe généralement leur émergence en 1984, lorsque Richard M. Stallman, alors chercheur au Massachusetts Institute of Technology, formalise la licence GPL ${ }^{(2)}$. En réalité, le logiciel libre n'aurait pu exister s'il ne s'était pas inscrit comme un héritage des coopérations des débuts de l'informatique. Il est alors courant de s'échanger des programmes et des codes sources ${ }^{(3)}$ entre chercheurs pour obtenir des remarques afin d'en améliorer la qualité, selon la même méthode que pour une publication universitaire s'échangeant entre pairs. Les premiers logiciels sont donc produits dans ce cadre de communautés non formalisées de partage (Levy, 2001; Perret, 2005).

La problématique marchande ne sera introduite qu'au milieu des années 70 , lors de l'apparition de la micro-informatique grand public. Il a fallu pour cela doter les logiciels des propriétés marchandes de la 
utilisateur. La règle d'accès doit être la même pour tous (le logiciel peut être payant, mais le point précédent permet à tout individu de le redistribuer gratuitement ensuite); - fraternité dans la réutilisation du code source. Tout développeur ayant modifié un code doit remettre I'intégralité de ses modifications dans la même licence. Ce procédé important, dit de copyleft, permet de conserver le code libre et en empêche toute appropriation.

(3) Qui constituent la matière première pour la production d'un logiciel.
(4) Sur les termes de marchand non marchand et lucratif : il est possible d'avoir des transactions marchandes non lucratives. On séparera donc la lucrativité et le caractère marchand ou non marchand de l'activité, le logiciel libre pouvant être marchand et non marchand.

(5) L'hybridation sera entendue ici comme désignant " un principe de transformation de modèles productifs au contact de systèmes sociaux et économiques différents de ceux sur lesquels ils ont pris leur essor " (Boyer, 1998, p. 12); l'importation de dispositifs d'autres systèmes renforce "l'efficacité $d u$ modèle existant et vice versa " (idem, p. 17). théorie économique (Varian, 1992), qu'ils n'avaient pas à l'origine: interdiction de partage, pour créer une rivalité dans l'usage, et dissimulation du code source, uniquement accessible en interne à l'entreprise commercialisant le logiciel, pour créer une exclusion d'accès au cycle productif. Une telle opération a choqué la communauté des producteurs au point qu'ils se lancent alors dans la réalisation du cadre permettant l'existence de ce que l'on appelle aujourd'hui le logiciel libre. Dans la fin du $\mathrm{XX}^{e}$ siècle dominée par la poussée néolibérale, son succès est un rare exemple de mouvement de "démarchandisation » d'un secteur. Un des paradoxes est que, de ce fait, bon nombre d'acteurs marchands vont, à partir de la seconde moitié des années 90 , organiser leur propre développement autour du logiciel libre, selon une formule d'hybridation tout à fait inattendue.

Pouvoir rendre compte de ce mouvement en termes d'analyse économique imposait de notre point de vue de s'écarter des catégories dominantes de biens (terme qui suppose que l'on entre dans un circuit marchand ou que le bien possède des mauvaises qualités marchandes, auquel cas on le qualifiera de bien public), de marché et d'externalités, fussent-elles positives ${ }^{(4)}$. Dans une première partie, on présente donc une approche théorique issue d'un séminaire pluridisciplinaire autour de l'économie patrimoniale: il s'agit de comprendre le jeu de formation en communautés et son corollaire, la constitution du logiciel en patrimoine commun. Dans une deuxième partie, on décrit le mouvement d'institutionnalisation du logiciel libre comme mouvement de patrimonialisation particulier, constitué dans une logique d'économie sociale. Dans une troisième partie, on précise deux éléments centraux dans la régulation de ce mouvement: la mise en place d'une règle de démembrement du droit de propriété en droits d'usage et droits de contrôle qui supposeront l'existence d'une communauté dédiée à la préservation de la logique d'économie sociale; l'émergence d'organisations dédiées à la production et développant chacune une spécificité quant à l'hybridation du marchand et du non-marchand ${ }^{(5)}$.

\section{Pour une approche patrimoniale du secteur des logiciels}

(6) Sur les aspects productifs très concrets des communautés fonctionnant en "dynamique servicielle ", on se permettra de renvoyer à " Instrument, activité et développement du pouvoir d'agir ", par Pierre Rabardel, et « Innovation et création de valeur dans les communautés d'action: les transactions communicationnelles symboliques ", par Manuel Zacklad, in Entre connaissance et organisation: l'activité. L'entreprise face au défi de la connaissance, sous la direction de Régine Teulier et
Dès lors que l'économie du logiciel quitte le public spécialisé capable d'échanger sur la création et l'amélioration des logiciels, elle connaît un changement dans la relation de service qui a présidé à la préhistoire de l'informatique. On peut alors envisager de séparer et de mettre en relation producteurs et utilisateurs dans un cadre marchand. Mais ce n'est pas la seule solution de coordination possible. Par ailleurs, toute solution nécessite, au-delà de l'offreur et du demandeur, l'engagement de communautés (communautés de producteurs, de marchands ou d'acteurs partageant les mêmes règles, communautés d'usagers capables de bénéficier d'effets réseaux par exemple) ${ }^{(6)}$. 
Philippe Lorino, éd. La Découverte, collection " Recherches ", 2005.

(7) Le rapport marchand s'entend au sens que lui donne la sociologie économique (Chantelat, 2002) de dépersonnalisation de la relation pour qu'elle ne laisse place qu'à la confrontation d'équivalents au sein d'un échange. Le rapport marchand est ce travail de dépersonnalisation toujours inachevé qui fait de la relation marchande une relation structurellement incomplète (Hodgson, 1999).

(8) Le rapport patrimonial peut être saisi à partir de quatre concepts articulés. Celui de patrimoine a ses trois niveaux d'expression: le privé, le collectif et le commun. Celui de communauté rappelle qu'il n'y a pas de patrimoine sans appartenance - ce qui est différent de la propriété. Celui d'identité renvoie à un impensé des économistes qui se targuent d'un choix rationnel du consommateur: dans l'expression des préférences sous contrainte des ressources rares, il est bien plus fondamental de décider de qui I'on veut être que de ce qu'on veut avoir, comme le rappellent Akerlof et Kranton (2002). Or, le patrimoine a plus à voir avec " l'être " qu'avec "l'avoir " (Fortunet, 2005). Le quatrième concept est celui de patrimonialisation, c'est-à-dire le mouvement par lequel un objet est élaboré en tant que ressource par un sujet individuel ou collectif, pour organiser son rapport au futur (Micoud, 2005).

(9) Voir Montgolfier J., Natali J.-M. (1987).

(10) Un exemple: si vous utilisez un document Word, celui-ci vous permet de communiquer avec une autre personne. Microsoft crée du lien, du bien commun, mais à l'intérieur de sa sphère privée.
Cela nous amène à mobiliser le cadre de l'économie du patrimoine, développé par un séminaire interdisciplinaire de l'université de Reims (Barrère et alii, édit., 2005; Nieddu, édit., 2004). En économie du patrimoine, on s'intéresse à la tension et aux effets de composition entre deux rapports sociaux aux logiques fondamentalement différentes, l'un étant le rapport marchand ${ }^{(7)}$, l'autre visant à préserver le groupe dans lequel peut se déployer l'action, que l'on a qualifié ailleurs de « rapport patrimonial » (Barthélemy, Nieddu, Vivien, 2006). Le premier est dominant dans nos sociétés, il a une efficacité, il produit dans certaines situations de la liberté, mais ne peut prétendre à fonder les groupes sociaux ${ }^{(8)}$.

Dans cette perspective, le patrimoine est un outil d'action sociale. Comme dit Olivier Godard (1990), ce n'est pas essentiellement du passé; il signifie que nous voulons que l'avenir contienne un certain nombre d'éléments sans lesquels nous estimons que la vie ne mérite pas d'être vécue. Le patrimoine est donc avant tout une prise du pouvoir sur l'avenir. En définissant la nature et la forme que prendra un patrimoine, on détermine quelle communauté va avoir le pouvoir d'agir sur les rapports entre les hommes: la communauté financière? la communauté des titulaires de droits de propriété ? la communauté industrielle fondée sur la coopération au travail? ou une communauté plus large, celle de l'ensemble des ayants droit, des générations futures? En décidant du périmètre de la communauté, on n'accordera pas aux personnes le même pouvoir d'agir (Nieddu, 2006).

Pourquoi ce détour de production par les patrimoines? Le secteur est marqué par deux modèles de l'économie de service. Le premier est fondé sur la relation client: il s'agit de l'insérer durablement dans la dépendance au marché en vue d'accumuler du patrimoine privé. Le second est le logiciel libre; il s'agit d'un patrimoine social (collectif ou commun, selon les formes ou les règles d'accès), au sens où c'est une ressource libre pour le groupe social considéré (Billaudot, 2004).

Dans la philosophie de Stallman ("free software, free society "), l'importance prise par l'informatique impose, si l'on souhaite rester libre, la formation de patrimoines communs. Cette remarque est d'autant plus importante que l'on a affaire à de très grandes organisations et donc que, topologiquement, le patrimoine commun peut être enchâssé à l'intérieur du patrimoine privé( ${ }^{(9)}$. Mais un avantage du logiciel libre sur le logiciel propriétaire tient au fait que l'on se trouve en économie des services. Dans celle-ci, "ce n'est plus tant l'action que l'interaction " qui est aujourd'hui source de productivité (Zarifian, 2006). Le problème est donc de fournir des ressources à l'interaction, à ce qui fait communauté. Or dans les expériences décrites, notamment celles des associations dans la troisième partie de ce texte, on retrouvera des stratégies bien connues en économie patrimoniale sous le nom de gestion patrimoniale négociée ${ }^{(10)}$. Celle-ci suppose la recherche d'un jeu de composition entre patrimoine privé, patrimoine collectif et patrimoine commun, acceptable par toutes les parties. 


\section{Le mouvement historique de patrimonialisation et d'hybridation marchand/non-marchand}

(11) On retiendra ici que le patrimoine collectif est - pour des raisons qui peuvent varier selon les situations - le support d'un groupe restreint, contrairement au patrimoine commun qui revendique dans sa qualification même une universalité. Pour des descriptions fines des processus de constitution des communautés, et des travaux récents qui qualifient ces communautés de "communauté de pratique ", voir Coris, 2004, de " communautés épistémiques ", voir Conein, 2004.

(12) « La communauté [...] du laboratoire d'IA s'était effondrée peu de temps auparavant. La plupart d'entre eux avaient été engagés par une nouvelle société [...], Symbolics, et ceux qui étaient restés ne parvenaient pas à maintenir la communauté. [...] Quand le laboratoire a, en 1982, choisi d'acheter un nouveau PDP-10, ses administrateurs ont décidé de remplacer ITS par le système de temps partagé de la société Digital, qui n'était pas libre... Les ordinateurs modernes d'alors, tels que le VAX ou le 68020, disposaient de leurs propres systèmes d'exploitation, mais aucun d'entre eux n'était un logiciel libre: il fallait signer un accord de nondivulgation pour en obtenir ne serait-ce que des copies exécutables. Cela signifiait que la première étape de l'utilisation d'un ordinateur était de promettre de ne pas aider son prochain. On interdisait toute communauté coopérative. La règle qu'édictaient ceux qui détenaient le monopole d'un logiciel propriétaire était "Qui partage avec son voisin est un pirate. Qui souhaite la moindre modification doit nous supplier de la lui faire" " (Stallman, 1998).

\section{D'une communauté d'experts aux patrimoines privés?}

Considérer les logiciels libres comme un patrimoine amène dans un premier temps à analyser la communauté fondatrice du mouvement (Stallman, 1998). Celle-ci est composée d'informaticiens, produisant pour eux-mêmes. C'est une population disposant d'un bon niveau de technicité et dont les demandes sont particulières. Chaque utilisateur a les capacités techniques pour devenir producteur. Ces premiers logiciels ne correspondent pas à des besoins grand public et leur utilisation reste difficile d'accès du fait de l'absence d'interface graphique. En revanche, le partage de la ressource se fait de façon spontanée au sein de la communauté, qui accumule ainsi un patrimoine collectif ${ }^{(11)}$. Comme le raconte Stallman (1998), de telles communautés s'effondrent avec l'émergence de logiciels propriétaires ${ }^{(12)}$.

Bill Gates (1976) prétendait justifier le passage du logiciel en logiciel propriétaire et donc en patrimoine privé par la nécessité de rémunérer les développeurs. Stallman propose un autre processus de patrimonialisation, dont le vecteur sera le copyleft. Stallman et Moglen (2005) considèrent ainsi rétrospectivement que "le mouvement du logiciel libre a tiré avantage des conditions sociales de son époque pour fonder son programme sur la création d'une nouvelle grande richesse grâce à de nouveaux systèmes de coopération, qui peut en retour être partagée pour augmenter cette nouvelle richesse, dans une boucle rétroactive positive ». En protégeant les logiciels qui seront alors qualifiés de libres, se met en place un processus de développement assurant une accumulation continue du patrimoine commun, ainsi qu' un rôle de préservation à l'encontre de l'opportunisme (le copyleft interdit toute appropriation sous une autre licence qui romprait l'accumulation du bien patrimonial).

\section{Logiciels libres et patrimoine commun contre logiciels propriétaires?}

L'effondrement des premières communautés a ainsi entrainé une recomposition du projet de quelques-uns des acteurs d'origine qui se sont demandé comment ils pourraient réécrire des programmes qui "permettraient de souder à nouveau une communauté " (Stallman, 1998, op. cit.). Le patrimoine est donc à la fois l'objectif à atteindre et la ressource pour créer la communauté.

La différence avec la période précédente réside de notre point de vue dans la volonté de la communauté d'origine de constituer et de qualifier légalement un patrimoine commun, ce qui nous amène à distinguer deux " cercles » de communautés, la communauté des développeurs en logiciels libres qui va se maintenir en tant que " communauté épistémique " (gestionnaires en patrimoine collectif) et celle des usagers, ayants droit 
(13) Il y a d'ailleurs recherche d'une reconnaissance institutionnelle de ce caractère de patrimoine commun par une demande en cours à I'Unesco pour reconnaître les logiciels libres comme patrimoine mondial de I'humanité (www.france.fsfeurope.org/ projects/mankind/mankind.fr.html). La réponse actuelle de I'Unesco est qu'il faut envisager cette question logiciel par logiciel. (en patrimoine commun) non seulement sur l'usage, mais aussi sur la conception des logiciels. Un tel modèle, s'il est effectivement nouveau dans certains secteurs, doit être vu à l'aune des habitudes de l'économie sociale, où les élus et les $A G$ de mutuelles ou d'associations ont un rôle de coordination entre producteur et usager, car l'usager a un droit de décision sur la production. Les demandes d'innovations ou de corrections de bugs ouvrent ainsi pour Eric von Hippel (2005) la voie vers une forme particulière d'innovation, qu'il nomme innovation ascendante. Celle-ci relève des interactions entre usagers et producteurs, et non plus d'un scénario « technologie push » où le producteur est juge des innovations qu'il estime intéressantes pour l'utilisateur (graphique 1).

Le patrimoine commun est donc le résultat et le moteur de l'existence du patrimoine collectif, en permettant la définition et l'expression de certains besoins basés sur la valeur d'usage et non sur la lucrativité. Comme on l'a vu, pour la communauté du libre, c'était l'objectif explicite de la création des logiciels libres ${ }^{(13)}$.

\section{La reconnaissance et la mobilisation du patrimoine commun par des acteurs marchands}

Si les logiciels libres relèvent d'une logique non marchande, ils acceptent l'introduction d'une dimension marchande, autorisée par la possibilité pour tous de redistribuer ces logiciels sous forme marchande et non marchande. Ce système va permettre à des entreprises privées de s'investir dans la production et la commercialisation de différentes façons. Des sociétés de services, le plus souvent de petite taille, reprennent des logiciels libres pour des adaptations ponctuelles à la demande de clients (Coris, 2004); d'autres laissent des personnels se mobiliser pour la communauté sur leur temps de travail; d'autres encore affectent des salariés en mission sur des dévelop-

\section{Graphique 1}

Interactions entre patrimoine commun et patrimoine collectif

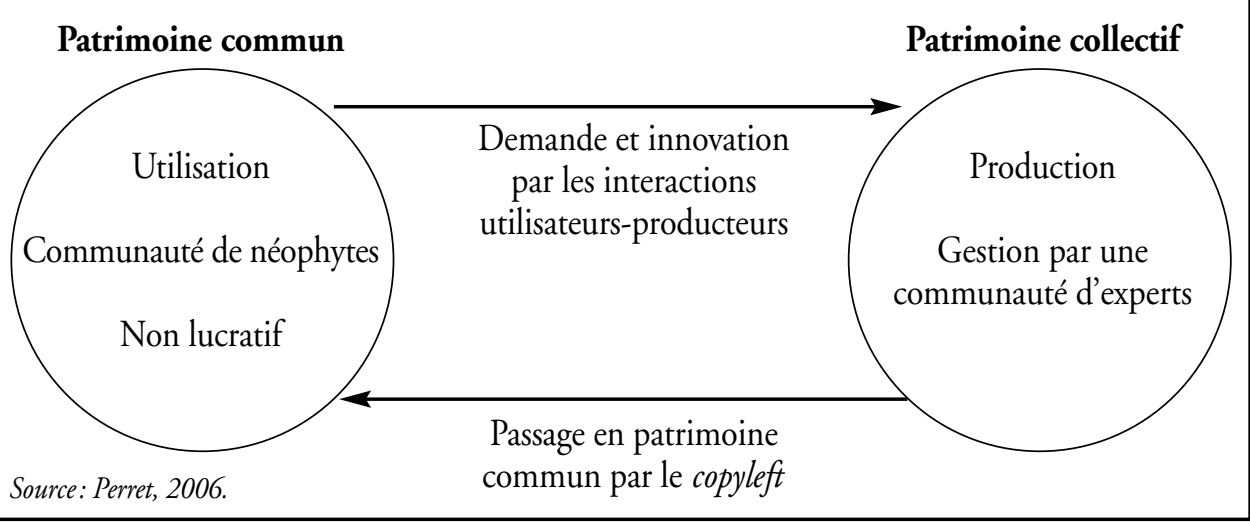


pements en cours. IBM déclare ainsi avoir atteint le nombre de mille salariés mobilisés selon ces deux formules. Enfin, certaines entreprises stimulent l'émergence de communautés spécifiques (Horn, Demazière, Zune, 2006). On assiste également, à un rythme soutenu, à un transfert de droits propriétaires en logiciels libres, soit de la part de sociétés concurrencées par des logiciels libres, soit de sociétés voulant s'ouvrir à des réseaux (Jullien, Clément-Fontaine, Dalle, édit., 2002). Des entreprises participent donc, pour des raisons qui leur sont propres, à la constitution en patrimoine commun, introduisant ainsi un rapport particulier au marché (graphique 2).

\section{Graphique 2}

Interactions entre les trois formes de patrimoine

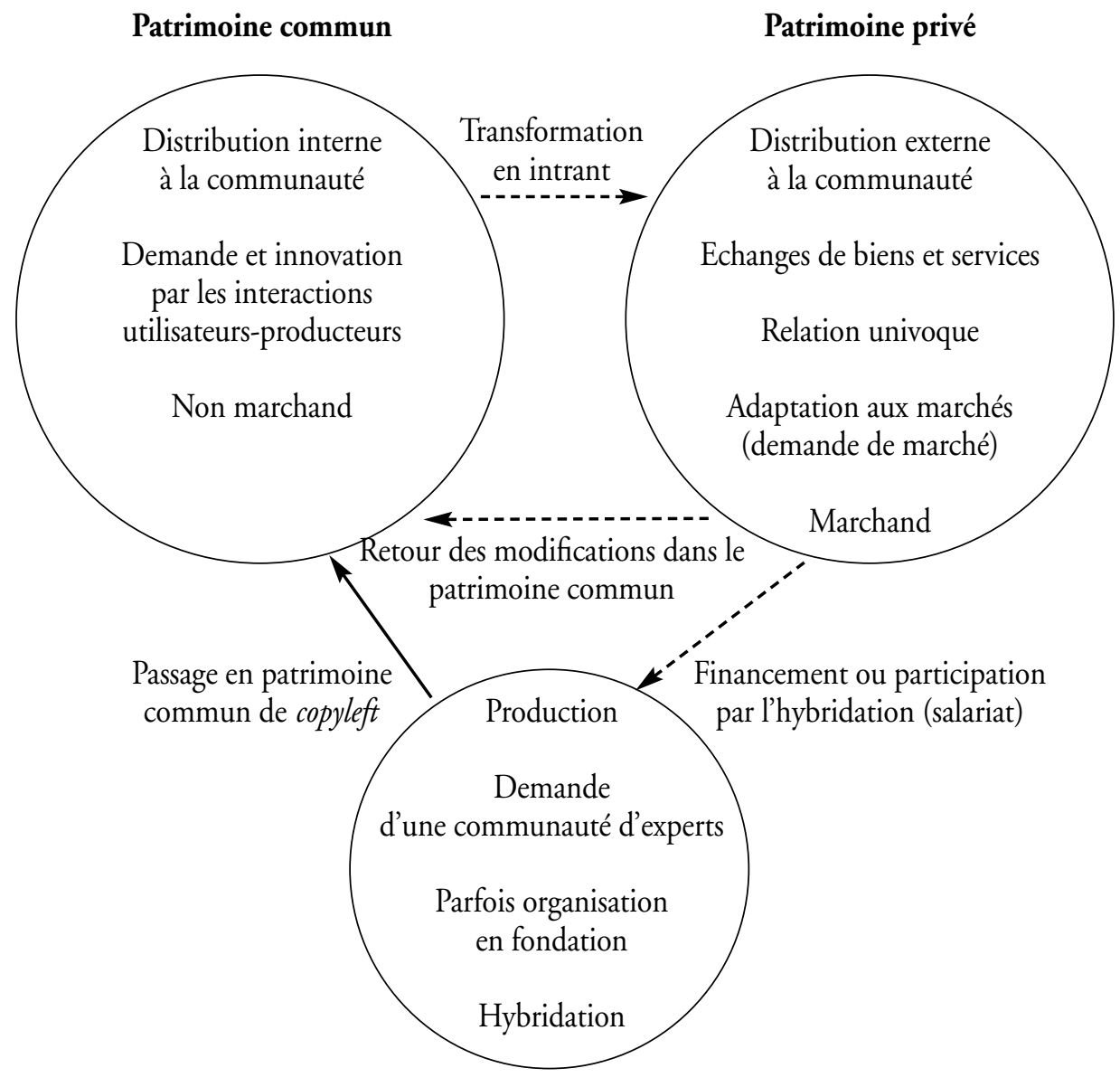

Source: Perret, 2006.

Patrimoine collectif 
Le patrimoine commun existe comme ressource, pouvant être adaptée aux besoins d'un marché. Mais, comme l'obligent les licences copyleft, les aménagements doivent revenir dans le patrimoine commun, alimentant ainsi le cercle vertueux mentionné par Stallman. Cependant, l'arrivée des firmes peut conduire à ne prendre en considération que la demande de marché, aux dépens du reste de la communauté. Des demandes pressantes en termes de délais contradictoires avec le bénévolat pourraient aussi amener les bénévoles à se désengager, entraînant une modification de la nature de la communauté de producteurs avec une forte composante de salariés délégués par les entreprises (Jullien, Zimmerman, 2005).

\section{La recherche de régulations de l'hybridation marchand/non-marchand}

(14) Un enjeu serait bien sûr de savoir s'il est possible de monter en généralité, en particulier en envisageant la possibilité de comparer à d'autres secteurs les solutions repérées (Prades, 2004b).

(15) S'opposant au courant de pensée de Hardin sur l'impossibilité d'une action collective, de nombreux auteurs, tel Ostrom (1990, 1999), ont présenté les fondements d'une approche institutionnaliste qui met l'accent sur les mécanismes de régulation, formels ou informels, qui gouvernent la viabilité des écosystèmes. Gouverner fait référence aux représentations des acteurs et se fonde sur un principe de négociation.

(16) « Une partie de la réponse tient certainement au fait qu'utiliser du logiciel n'en décroît en rien la valeur. En fait, le fait qu'un logiciel à sources ouverts soit largement utilisé tend à en augmenter la valeur, puisque les utilisateurs contribuent par leurs propres corrections et ajouts (par des patches au code). Dans ces communs inversés, l'herbe repousse plus haut quand elle est broutée. " Raymond Eric S., The Magic Cauldron, juin 1999, traduit en juillet: www.linux-france.org/article/these/ magic-cauldron/magic-cauldronfr-5.html.
Le débat sur les logiciels libres nous intéresse du fait de la capacité qu'ont eue des acteurs, à un moment donné, de trouver des solutions de coopération économique reposant sur l'économie sociale; la question est alors de savoir s'il est possible d'identifier analytiquement les moyens utilisés pour stabiliser en dynamique ces solutions de coopération et leur rapport au marché ${ }^{(14)}$.

On reviendra donc d'abord sur le premier type de régulation de ces rapports entre marché et patrimoines communs et collectifs avec l'instauration en 1984 du système du copyleft et d'une association dédiée à sa protection. Un second type de régulation, qui nous semble encore instable, émerge à la fin des années 90. L’arrivée de firmes puissantes dans la production de logiciels libres modifie brutalement les rythmes de développement du patrimoine collectif et in fine l'organisation des communautés supports; celles-ci prennent la forme d'associations à but non lucratif visant explicitement à réguler des aspects spécifiques de l'hybridation entre marchand et non-marchand (Perret, 2004, 2005; O’Mahony, 2005).

\section{Sur la nature de l'innovation juridique pour réguler l'accès et la diffusion du patrimoine}

L'argument bien connu contre les solutions de coordination coopérative tient au problème de la " tragédie des communs ». La licence GPL est une version moderne des critiques de la métaphore de cette tragédie: les communs n'étaient pas, contrairement à ce que prétend Hardin, des biens libres, mais ils étaient soumis à des règles d'usage parfois extrêmement contraignantes; c'est le recul de ces règles ou la dislocation des communautés supports qui, de fait, génère les "tragédies " ${ }^{(15)}$. Néanmoins, on ne peut partager le point de vue des auteurs qui, tel Raymond (1999), expliquent de façon téléologique l'existence de la licence GPL par son efficacité $^{(16)}$. En quelque sorte, suivant en cela une explication à la North (1990) sur la justification rationnelle d'institutions, le marché aurait sélectionné cette forme institutionnelle comme plus efficace pour la production du bien. 
(17) Voir Transcript of Richard Stallman at the $5^{\text {th }}$ International GPLv3 Conference, $21^{\text {st }}$ November 2006 : www.france.fsfeurope.org/ projects/gplv3/tokyo-rms-transcript.

(18) La licence GPL elle-même n'est pas un « produit libre " au sens où, contrairement au logiciel, elle n'est pas modifiable par toute personne souhaitant le faire, mais protégée par I'utilisation de cadres juridiques classiques.

(19) Ces associations sont sous statut de "foundation " américaine de type 501(c)(3), correspondant aux associations françaises. Les structures de type 501(c) désignent un ensemble d'organisations bénéficiant de certaines exemptions fiscales. Ces structures 501 (c) se décomposent en de nombreuses catégories. Les fondations de logiciels libres se retrouvent dans la troisième et y côtoient notamment des organisations humanitaires comme la Croix-Rouge américaine. (20) Voir les statuts sur les sites des fondations indiquées en bibliographie.
En réalité, d'une part, la licence GPL aurait très bien pu ne pas émerger et nécessite un effort constant d'entretien: la communauté autour de Stallman prépare la troisième version de GPL pour répondre aux risques liés aux évolutions du secteur ${ }^{(17)}$. D'autre part, il faut la concevoir dans le système de droits formels et informels qu'elle forme avec les règles des communautés supports. La logique de préservation et de développement du patrimoine collectif conduit à une combinaison originale des règles de gestion du patrimoine commun (la licence GPL donne accès à tous) et du patrimoine collectif (la communauté des développeurs a besoin d'une organisation et donc de règles de production). Notre point de vue est qu'à cet endroit il est nécessaire de revenir au régime de droits de propriété en nous inspirant de la méthode d'anthropologie juridique de Le Roy (1999), qui pose le principe du démembrement dans la réalité de droits de propriété présentés sous l'unité théorique de l'« usus, abusus, fructus ». La licence fournit un droit d'accès, alors que les évolutions du logiciel (le droit d'intégrer les altérations du logiciel) sont " filtrées " par la communauté de développeurs. Cette dissociation droits d'accès/droits de contrôle, que l'on retrouve avec la Free Sofware Foundation pour la gestion de la licence GPL, est le support de l'articulation droits-devoirs qui selon les spécialistes est au fondement de la protection des biens communs ${ }^{(18)}$. Il est alors possible de faire exister un autre système sécurisant la préservation de certains objets que le système des droits de propriété marchands.

\section{L'émergence de structures d'économie sociale pour réguler l'hybridation marchand/non-marchand}

De 1984 jusqu'au début des années 90, la production des logiciels libres va essentiellement être le fait d'une communauté composée d'individus, à majorité bénévole; ceux développant un aspect marchand le font comme moyen de subsistance plus que pour la recherche de lucrativité. L'entrée de firmes privées amène l'émergence d'associations ${ }^{(19)}$, pour lesquelles nous avons cherché à identifier les tensions qu'elles cherchaient à réguler dans le rapport marchand/non-marchand. Ces associations possèdent majoritairement un statut de fondation californienne à but non lucratif de type 501(c)(3) ${ }^{(20)}$. On observe l'intensité de ce mouvement sur la période 19982000, avec notamment six créations majeures en 2000 (O'Mahony, 2005, op. cit.).

Nous étudierons plus spécifiquement trois associations. Gnome se crée pour réguler l'intervention d'entreprises concurrentes entre elles intervenant dans la production d'un même logiciel libre, Open Office, à l'initiative d'une entreprise souhaitant passer à un mode de production coopératif, et Mozilla, pour intégrer en son sein une régulation salariale.

\section{L'association comme institution de régulation des relations entre acteurs marchands: le cas de la Fondation Gnome}

La Fondation Gnome prend en charge le développement du logiciel éponyme, l'une des interfaces graphiques majeures du système d'exploitation 
(21) Le paradoxe étant que les mêmes systèmes peuvent être téléchargés gratuitement. Ces entreprises peuvent ainsi bénéficier du travail en libre de la communauté. (22) "With the emergence of a Gnome industry, and as corporate partners are embracing Gnome, the number of hackers who are paid to work full-time on Gnome has grown dramatically, in addition to the hundreds of volunteer hackers in the community. [...] We need a more structured environment to smoothly integrate new citizens into the community. The Gnome Foundation will provide this support. The Foundation will also provide a place to resolve the inevitable conflicts that arise in a diverse community. Equally important, the Foundation can voice the decisions and positions of the Gnome project, and, therefore, can act as a liaison with the press and corporations who want to be involved with Gnome. ” (http://foundation. gnome.org/about/charter.)

(23) Interview de Mathieu Lecage, membre du bureau de la Fondation Gnome, par F. Perret (Perret, 2004). (24) Une décision du board of directors peut cependant être annulée par un référendum demandé par au moins $10 \%$ des membres.

(25) Les entreprises intéressées dans le logiciel peuvent cependant siéger dans l'advisory board en payant des droits d'entrée. Dans ce bureau, qui ne peut prendre aucune décision, elles peuvent une fois par an discuter des évolutions du logiciel et obtenir un contact direct avec les développeurs élus. Adhérer à ce conseil permet aussi de bénéficier d'une image positive auprès des membres de la communauté, qui sont aussi souvent des clients. Les entreprises adhérant à l'advisory board de Gnome sont parmi les plus importantes en informatique, notamment IBM, HP, Novell, Sun, Mandriva, Red Hat... L'existence de ce lieu d'échange permet de contenir les pressions que pourraient être amenées à faire les entreprises. II leur donne un cadre où elles peuvent soumettre des idées tout en sachant que les décisions ne leur reviennent pas.
GNU/Linux. Ce logiciel est utilisé par un grand nombre d'acteurs lucratifs, notamment ceux commercialisant des " distributions ", c'est-à-dire des ensembles coordonnés de logiciels formant un système d'exploitation (Novell, Red Hat, Mandriva....) ${ }^{(21)}$. La création de la fondation répond à l'objectif de mieux coordonner un nombre devenu plus important de développeurs, pour certains venant d'entreprises. Elle aura ainsi la charge de gérer le patrimoine commun et de représenter la communauté(22).

L'histoire de Gnome, créée en opposition à une autre interface graphique pour GNU/Linux non entièrement libre à cette époque (KDE), peut permettre de comprendre les principes adoptés par la fondation: avant la constitution de la fondation, les décisions stratégiques étaient prises par une seule personne (Miguel de Icaza, l'initiateur de Gnome), avec éventuellement des discussions privées avec quelques personnes. Pour que la fondation soit acceptée par la totalité des développeurs participant au projet, le choix s'est porté vers un mode de désignation démocratique. La charte prévoit explicitement que "la fondation doit être indépendante des influences des organisations extérieures et des entreprises, et aucune entité seule ne doit pouvoir diriger Gnome pour ses propres fins" (traduit par nous). L'ancien système où une personne décidait seule, après discussions avec les entreprises, occasionnait des tensions avec les autres développeurs ${ }^{(23)}$.

Des élections mondiales sont organisées chaque année pour désigner les onze membres du "board of directors » chargé de prendre toutes les décisions, notamment en fixant les objectifs et la vision à long terme pour le logiciel ${ }^{(24)}$. Toutes les discussions sont publiques et archivées. La fondation apparait pour l'essentiel comme un moyen d'encadrer la pratique des entreprises souhaitant participer au développement. En effet, les contributions ne sont pas considérées par la fondation comme venant d'entreprises, mais comme provenant d'un individu qui peut éventuellement être salarié d'une entreprise. Mais celle-ci doit accepter de ne pas peser sur les décisions stratégiques. Si elle souhaite une amélioration dans le logiciel, elle peut demander à l'un de ses salariés de la créer pour qu'il puisse ensuite la soumettre à la fondation, qui demeure seule juge de l'intérêt d'intégrer un développement additionnel au programme. Il est courant que des entreprises en concurrence sur la distribution ou les services d'un logiciel coopèrent dans la production de celui-ci (une centaine de salariés de ces entreprises travaillent ensemble à temps plein sur Gnome).

La constitution d'un espace de socialisation du travail salarié d'entreprises privées différentes s'accompagne de dispositifs permettant d'éviter la prise de contrôle de la fondation: les statuts interdisent que plus de $40 \%$ des élus travaillent pour une même entreprise ${ }^{(25)}$.

\section{Une association pour intégrer le rapport salarial: le cas de la Fondation Mozilla}

La Fondation Mozilla se crée en juillet 2003 lorsque Netscape, alors racheté par AOL, décide d'abandonner le développement de son navigateur 
(26) Le logiciel Mozilla est issu de la crise de Netscape, Iorsque Microsoft intègre Internet Explorer. Netscape passe alors en logiciel libre, mais son code source s'avérera de mauvaise qualité. Netscape va financer le développement du nouveau logiciel dans un statut de logiciel libre.

(27) Ses ressources proviennent d'accords signés avec des moteurs de recherche, notamment Google, pour figurer dans les liens de recherche par défaut du navigateur Firefox. La fondation touche ainsi une somme fixe à laquelle s'ajoute un pourcentage sur les publicités vues par les internautes lorsqu'ils arrivent sur le site du moteur de recherche par ces liens.

(28) Mitchell Baker, The Mozilla Foundation: Achieving Sustainability, January 2, 2007, http://weblogs. mozillazine.org/mitc h e I I / a r c h i ves / 2007/01/the_mozilla_foundation

(29) Sun est l'acronyme de Stanford University Network. L'entreprise fut à l'origine créée par des universitaires de Stanford pour défendre Unix face à la montée du système d'exploitation de Microsoft. En parallèle de la version libre, Sun a choisi de différencier sa propre version d'Open Office, appelée Star Office, en lui adjoignant divers logiciels propriétaires (comme un correcteur grammatical) et des services d'assistance.
Internet ${ }^{(26)}$. AOL aide au démarrage en effectuant un don de 2 millions de dollars sur deux ans. En 2005, la fondation a réalisé un chiffre d'affaires combiné de 52,9 millions de dollars (pour 5,8 millions de dollars en 2004), issus pour l'essentiel du partage des recettes publicitaires de Google ${ }^{(27)}$. Avec des coûts de fonctionnement 2005 de 3 millions de dollars, la fondation réalise un bénéfice net de 49,9 millions de dollars. Nous retrouvons donc ici une forme classique de financement par tiers d'une structure non marchande.

La particularité et la raison d'être de la Fondation Mozilla, c'est d'ajouter un complément à la formule vue précédemment de bénévoles et de salariés d'entreprises dont le travail est socialisé. La fondation salarie directement une partie des développeurs travaillant sur les différents logiciels qu'elle a en charge (Firefox, Thunderbird, Sunbird, Bugzilla, etc.). Alors qu'elle ne comptait que trois salariés à sa création, la fondation emploie maintenant soixante-dix personnes et ce chiffre devrait continuer à progresser grâce aux bénéfices réalisés. Il s’agit bien d'une gestion de la relation salariale. La présidente Mitchell Baker déclare ainsi : "Notre stabilité financière nous permet désormais d'attirer et de conserver des talents d'envergure internationale, des gens capables de tourner le dos à l'univers des start-up et de leurs stock-options, et préférant soutenir le choix et l'innovation dans l'Internet pour le plus grand bénéfice de tous ${ }^{(28)}$."

L'enjeu semble donc bien d'attirer des développeurs de haut niveau pour les dédier à temps plein à la fondation. Cela supposait d'introduire une nouvelle possibilité, en organisant pour le travail un espace marchand non lucratif au sein de la communauté; et bien sûr, pour se financer, d'être capable de recueillir des revenus rendant solvable l'activité.

\section{L'abandon du logiciel propriétaire pour le logiciel libre: le cas d'Open Office}

La constitution du logiciel libre en norme amène les entreprises à l'intégrer dans leur propre stratégie. Le cas d'Open Office montre comment une entreprise peut effectuer un passage du logiciel propriétaire vers le logiciel libre ${ }^{(29)}$. L'argument principal donné par Open Office est que la constitution d'une communauté donne une efficacité plus grande grâce au développement coopératif qu’elle permet. Aujourd'hui, Sun déclare ne représenter que la moitié du travail effectué sur le code à ce jour (par une centaine de développeurs) et recevrait l'aide d'environ soixante-dix mille personnes toutes tâches confondues (développement, marketing, traduction, documentation, etc.). Cette part encore relativement importante s'explique par des problèmes de lisibilité du code source (dix millions de lignes de code) des logiciels de taille importante, d'autant plus pour une personne extérieure (ce qui n'avait pas été prévu dans le cadre du logiciel propriétaire). Une partie du travail consiste donc à créer de la documentation sur le fonctionnement du code. Néanmoins, il faut noter que Sun Microsystem se trouvait devant l'alternative d'abandonner cette suite bureautique, concurrente battue par Microsoft Office, ou de la libérer. Il n'y avait donc pas à renoncer 
(30) Voir "Blender, un modèle économique atypique ", 2003, www.logiciellibre.net/2003/ news20031210.php. à la rente que procure un logiciel propriétaire. Cela illustre l'attractivité d'une formule de "démarchandification", en particulier lorsque les difficultés économiques apparaissent et qu'elle devient la solution pour ne pas perdre l'effort productif antérieur (nous dirions dévaloriser le patrimoine antérieur accumulé, en le faisant passer d'un statut privé à un statut de patrimoine commun). C'est un scénario bien connu des praticiens de l'économie sociale qui voient des acteurs se tourner vers des statuts d'économie sociale tels que les Scop, lors de périodes de difficultés. Ce mouvement a pu être observé pour d'autres entreprises du secteur informatique, telles que Blender en $2002^{(30)}$.

\section{Conclusion}

Les trois communautés de logiciels libres (Mozilla Firefox, Open Office, Gnome), dont nous avons décrit rapidement les raisons d'existence, ont énormément évolué. Mais leurs caractéristiques montrent la persistance des trois types de patrimoines articulés - communs, collectifs ou privés -, au sens où se maintiennent des activités marchandes à partir de l'espace commun qu'elles structurent. Le succès même du copyleft va provoquer une évolution importante de la production des logiciels libres. L'arrivée massive d'acteurs marchands, qu'ils soient des sociétés de développement de logiciels (Red Hat, Novell...), puis des sociétés de services (SSLL) ou, enfin, des fabricants de matériels (IBM, Apple...), amène les communautés à des réflexions sur les moyens de pérenniser leurs valeurs de coopération et de partage du savoir.

Loin d'être linéaire et spontanée, la croissance des logiciels libres a donc été permise par la "main visible » de certains acteurs se comportant comme de véritables entrepreneurs sociaux. Deux clés institutionnelles doivent être retenues: l'existence de règles portées par des cadres juridiques qui peuvent les remettre en cause et l'existence d'associations. Mais l'un des moteurs qui nous semblent avoir guidé certains mouvements de passage du marchand au non-marchand a été le refus de voir la concurrence entre firmes venir détruire la valeur sociale crée précédemment. La solution du logiciel libre préservait alors des possibilités de jeux ultérieurs de conversion entre patrimoines communs et privés. Cela explique en partie l'investissement de firmes dans la transition d'une partie de la production vers l'économie sociale, tout en maintenant leur activité lucrative.

L'économie sociale apparaît alors comme un dispositif au cœur de la régulation économique du secteur, car elle met en forme des règles d'articulation du marchand et du non-marchand. Nous constatons que le résultat de ces régulations est non seulement la préservation et l'extension du patrimoine commun que constituent les logiciels libres, mais aussi le fait qu'elles influent sur l'ensemble du marché des logiciels, libres ou non, en obligeant les acteurs lucratifs à se repositionner et à faire évoluer leurs stratégies pour y intégrer une part de non-marchand. 


\section{Bibliographie}

Akerlof G., Kranton R. (2002), « Identity and Schooling: Some Lessons for the Economics of Education ", Journal of Economic Literature, volume 40(4), p. 1167-1201.

Barrère C., Barthelemy D., Nieddu M., Vivien F.-D. (2005), Réinventer le patrimoine: de la culture à l'économie, une nouvelle pensée $d u$ patrimoine? L'Harmattan, Paris.

Barrère C. (2003), « L'apport d'une problématique économique du patrimoine à l'analyse de la régulation institutionnelle ", IIIs journées d'étude "Approches économiques et pluri-disciplinaires du patrimoine ", http://helios.univ-reims.fr/Labos/ ESSAI/patrimoine/barrere.pdf, Reims, juin.

Barthélémy D., Nieddu M., Vivien F.-D. (2006), "Pour une refondation critique de la notion de patrimoine ", La Pensée, n 347, p. 109-118. Billaudot B. (2004), in Nieddu M. (édit.), "Patrimoines, ordres et dynamique du capitalisme ", Géographie, Economie, Société, vol. 6, no 3, p. 237-352.

Boyer R. (1998), « Hybridation et modèle productif: géographie, histoire et théorie ", Actes de Gerpisa, n' 24, p. 7-50.

Chantelat, P., (2002), « La nouvelle sociologie économique et le lien marchand: des relations personnelles à l'impersonnalité des relations ", Revue française de sociologie, 4363, 2002, p. 521-556.

Conein B. (2004), Communauté épistémique et réseaux cognitifs: coopération et cognition distribuées, Freescape, www.freescape.eu.org/biblio/ article.php3?id_article=176.

Coris M. (2004), « Le logiciel libre: émergence et hybridation d'une alternative productive ", thèse de doctorat en sciences économiques, université Montesquieu-Bordeaux IV, décembre. Coris M., Lung Y. (2005), « Les communautés virtuelles: la coordination sans proximité? Les fondements de la coopération au sein des communautés du logiciel ", Revue d'économie régionale et urbaine, $\mathrm{n}^{\circ}$ 2005-3, p. 397-420.
Draft Charter for the GNOME Foundation (2000), http://foundation.gnome.org/about/charter.

Fortunet F. (2005), « Patrimoine et identité: approches juridiques ", in Barrère C., Bathélémy D., Nieddu M., Vivien F.D. (édit.), Réinventez le patrimoine, de la culture à l'économie, une nouvelle pensée du patrimoine? L'Harmattan, Paris, p. 75-85.

Fondation Gnome, http://foundation.gnome.org. Fondation Mozilla, www.mozilla.org/foundation. Fondation OpenOffice, http://fr.openoffice.org/ about-governance.html.

Gates B. (1976), « Lettre ouverte aux "Hobbyistes" ", www.freescape.eu.org/biblio/ article.php3?id_article $=155$.

Godard O. (1990), «Environnement, modes de coordination et systèmes de légitimité: analyse de la catégorie de patrimoine naturel ", Revue économique, vol. 41, $\mathrm{n}^{\circ}$ 2, mars, p. 215-241.

Hodgson, G. M. (1999) Evolution and Institutions, on Evolutionary Economics and the Evolution of Economics, Edward Elgar, London. Horn F., Demazière D., Zune M. (2006), « La dynamique de développement des communautés du logiciel libre: conditions d'émergence et régulations des tensions ", Terminal, Technologie de l'information, Culture et Société, no 97-98.

Jullien N., Zimmerman J.-B. (2005), "Peut-on envisager une écologie du logiciel libre favorable aux nuls?", Cahier de recherche du Marsouin, $\mathrm{n}^{\circ} 9$.

Jullien N., Clement-Fontaine M., Dalle J. M. (2002), Nouveaux modèles économiques, nouvelle économie du logiciel, rapport final du projet RNTL, www-eco.enst-bretagne.fr/ Etudes-projets/RNTL.

Le Roy E. (1999), Le jeu des lois, une anthropologie "dynamique " du droit, LGDJ, Paris.

Levy S. (2001), Hackers: Heroes of the Computer Revolution, Penguin, New York.

Micoud A. (2005), " La patrimonialisation: redire ce qui nous relie? " in Barrère C., Bathélémy D., Nieddu M., Vivien F.-D. (édit.), Réinventez le patrimoine, de la culture à 
l'économie, une nouvelle pensée du patrimoine? L'Harmattan, Paris, p. 81-97.

Montgolfier J., Natali J.-M. (1987), Le patrimoine du futur, approches pour une gestion patrimoniale des ressources naturelles, Economica, Paris.

Nieddu M. (2006) «Revisiter l'économie du service à partir de la théorie économique du patrimoine ", colloque de Cerisy, L'économie des services pour un développement durable, 14-20 juin 2006, à paraître.

Nieddu M. [édit.] (2004), « Patrimoines, ordres et dynamique du capitalisme ", numéro thématique de Géographie, Economie, Sociétén ${ }^{\circ}$ 3/2004. North D. C. (1990), Institutions, Institutional Change and Economic Performance (Political Economy of Institutions and Decision), Cambridge University Press, Cambridge.

O’Mahony S. (2005), « Non-Profit Foundations and Their Role in Community-Firm Software Collaboration ", in Joe Feller, Brian Fitzgerald, Scott Hissam and Karim Lakhani (eds.), Perspectives on Free and Open Source Software, MIT Press, Cambridge.

Ostrom E. (1990), Governing the Commons, Cambridge University Press, Cambridge.

Ostrom E. (1999), "Coping with Tragedies of the Commons ", American Review of Political Science, vol. 2, juin, p. 493-535.

Perret F. (2006), « Patrimonialisation et droits de propriété: le cas des logiciels libres", $V^{e s}$ journées d'étude "Approches économiques et pluridisciplinaires du patrimoine, régulations, institutions et patrimoines, juin.

Perret F. (2005), « Les interactions économiques et sociales entre activités marchandes et non marchandes: le cas des logiciels libres ", mémoire de DEA de sciences économiques, université Reims Champagne-Ardenne, juin.

Perret F. (2004), "Les logiciels libres: un modèle pour un nouveau mode de production non marchand? ", mémoire de maîtrise de sciences économiques, université Reims Champagne-Ardenne, juin.
Prades J. (2004a), « Economie solidaire et technologies de l'information, une mise en perspective ", Terminal, Technologie de l'information, Culture et Société, $\mathrm{n}^{\circ} 30$.

Prades J. (2004b), "Community Development Corporations et logiciels libres: une anthropologie comparée des pratiques coopératives ", Terminal, $\mathrm{n}^{\circ} 91$.

Rabardel P. (2003), «Instrument, activité et développement du pouvoir d'agir ", in Entre connaissance et organisation: l'activité. L'entreprise face au défi de la connaissance, sous la direction de Régine Teulier et Philippe Lorino, éd. La Découverte, collection "Recherches ", 2005, Paris. Raymond E. (1999), The Magic Cauldron, www.linux-france.org/article/these/ magic-cauldron/magic-cauldron-fr.html.

Stallman R. (1984), The GNU manifesto, www.gnu.org/gnu/manifesto.html.

Stallman R. (1998), The GNU Project, www.gnu.org/gnu/thegnuproject.html.

Stallman R. et Moglen E. (2005), GPL Version 3: les coulisses de l'adoption, www.fsf.org/ licensing/essays/fr/gpl3-background.fr.html.

Stallman R. (2006), Transcript of Richard Stallman at the $5^{\text {th }}$ International GPLv3 Conference, $21^{\text {st }}$ November 2006, www.france.fsfeurope.org/ projects/gplv3/tokyo-rms-transcript.en.html. Varian H.-R. (1992), Introduction à la microéconomie, De Boeck, Bruxelles.

Von Hippel E. (2005), « Democratizing innovation ", MIT Press, Cambridge.

Zacklad M. (2005), « Innovation et création de valeur dans les communautés d'action : les transactions communicationnelles symboliques ", in Régine Teulier et Philippe Lorino (dir.), Entre connaissance et organisation: l'activité. L'entreprise face au défi de la connaissance, éd. La Découverte, collection "Recherches ", Paris.

Zarifian P. (2006), « Histoire et enjeux du service durable », intervention réalisée le 15 juin 2006 dans le cadre du colloque Cerisy intitulé L'économie des services pour un développement durable, http://perso.orange.fr/ philippe.zarifian/page152.htm. 